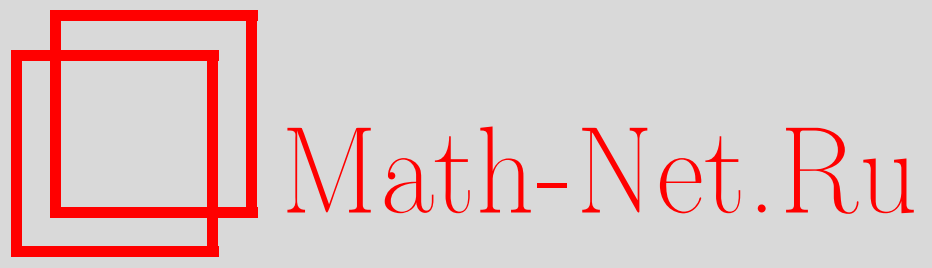

А. П. Шашкин, Асимптотическая нормальность оценок с локальным усреднением для слабо зависимых случайных полей, Теория вероятн. и ее примен., 2014, том 59, выпуск 3, 603-613

DOI: https://doi.org/10.4213/tvp4586

Использование Общероссийского математического портала Math-Net.Ru подразумевает, что вы прочитали и согласны с пользовательским соглашением http://www . mathnet.ru/rus/agreement

Параметры загрузки:

IP : 54.197 .217 .227

26 апреля 2023 г., 18:18:07

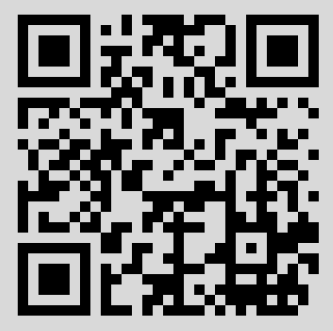




\title{
АСИМПТОТИЧЕСКАЯ НОРМАЛЬНОСТЬ ОЦЕНОК С ЛОКАЛЬНЫМ УСРЕДНЕНИЕМ ДЛЯ СЛАБО ЗАВИСИМЫХ СЛУЧАЙНЫХ ПОЛЕЙ
}

\begin{abstract}
Рассматривается семейство оценок долгосрочной дисперсии стационарного случайного поля, обладающего свойством слабой зависимости, более общим, чем положительная или отрицательная ассоциированность. Для оценок с локальным усреднением доказана асимптотическая нормальность, которая при некоторых дополнительных условиях позволяет строить асимптотические доверительные интервалы для неизвестной долгосрочной дисперсии. Получена также функциональная центральная предельная теорема.
\end{abstract}

Ключевые слова и фразы: слабая зависимость, ассоциированные случайные поля, долгосрочная дисперсия, доверительные интервалы.

Исследование асимптотического поведения слабо зависимых случайных процессов и полей - активно развивающаяся тема в современной теории вероятностей. Среди изучаемых в ней стохастических систем важное место занимают ассоциированные случайные поля и их обобщения. Напомним некоторые моменты.

О п р е д е л е н и е 1 (см. [10]). Случайное поле $X=\left\{X_{j}, j \in \mathbf{Z}^{d}\right\}$ называется ассоциированньл, если

$$
\operatorname{cov}\left(f\left(X_{i_{1}}, \ldots, X_{i_{n}}\right), g\left(X_{j_{1}}, \ldots, X_{j_{n}}\right)\right) \geqslant 0
$$

для любого $n \in \mathbf{N}$, любой пары неубывающих (покоординатно) ограниченных борелевских функций $f, g: \mathbf{R}^{n} \rightarrow \mathbf{R}$ и любых $i_{1}, \ldots, i_{n}, j_{1}, \ldots, j_{n} \in \mathbf{Z}^{d}$. Поле называется слабо (или положительно) ассочиированным, если оно удовлетворяет приведенному выше определению при дополнительном требовании, чтобы множества $\left\{i_{1}, \ldots, i_{n}\right\}$ и $\left\{j_{1}, \ldots, j_{n}\right\}$ не пересекались, и отрицательно ассоииированньл, если при том же дополнительном требовании ковариация в (1) неположительна.

Семейство, состоящее из независимых случайных величин, ассоциированно; многие примеры положительной и отрицательной ассоциированности возникают в статистике, математической физике, теории случайных мер и графов. Известны условия, обеспечивающие ассоциированность гауссовских, устойчивых, диффузионных процессов. Разнообразные предельные теоремы (сходимость к нормальному закону, принципы инвариантности, законы повторного логарифма и т.д.) для ассоциированных случайных полей и их обобщений получали Ч. Ньюмен, Т. Биркел, А. В. Булинский, Дж. Руссас, К.-М. Шао, Т. Кристофидес и многие другие ученые, см. монографии [4], [15] и там же библиографию.

Хорошо известно, что при исследовании асимптотического поведения ассоциированных и родственных им случайных систем большое значение имеет сходимость ряда $\sum_{j \in \mathbf{Z}^{d}} \operatorname{cov}\left(X_{0}, X_{j}\right)$ (условие конечной восприимчивости). Так, в случае стационарного в широком смысле ассоциированного случайного поля числа

$$
u_{r}=\sum_{j:\|j\|_{\infty} \geqslant r} \operatorname{cov}\left(X_{0}, X_{j}\right), \quad r \in \mathbf{N},
$$

(здесь $\|\cdot\|_{\infty}$ - максимальная норма в $\mathbf{R}^{d}$ ) называют коэффициентами Кокса-Гримметта, и, как правило, определенная скорость сходимости этих коэффициентов к нулю

* Московский государственный университет им. М. В. Ломоносова, механикоматематический факультет, Москва, Россия; e-mail: ashashkin@hotmail.com

1) Работа выполнена при поддержке РФФИ, проект № 13-01-00612. 
при $r \rightarrow \infty$ наряду с требованиями к моментам обеспечивает справедливость тех или иных предельных теорем. В 2001 г. А.В.Булинским и Ш. Сюке [9] предложено более общее условие слабой зависимости случайного поля, охватывающее как положительно, так и отрицательно ассоциированные поля (при условии, что они квадратично-интегрируемы и обладают свойством конечной восприимчивости).

О п р е д е л е н и е 2 (см. [9]). Случайное поле $X=\left\{X_{j}, j \in \mathbf{Z}^{d}\right\}$ называется $(B L, \theta)$-зависимьл, если существует последовательность $\left\{\theta_{r}, r \in \mathbf{N}\right\}$ такая, что

$$
\left|\operatorname{cov}\left(f\left(X_{i_{1}}, \ldots, X_{i_{m}}\right), g\left(X_{j_{1}}, \ldots, X_{j_{k}}\right)\right)\right| \leqslant \operatorname{Lip}(f) \operatorname{Lip}(g) \min \{m, k\} \theta_{r}
$$

для любой пары липшицевых функций $f: \mathbf{R}^{m} \rightarrow \mathbf{R}, g: \mathbf{R}^{k} \rightarrow \mathbf{R}$, и любых непересекающихся конечных множеств $\left\{i_{1}, \ldots, i_{m}\right\},\left\{j_{1}, \ldots, j_{k}\right\} \subset \mathbf{Z}^{d}$. Здесь $r-$ расстояние в норме $\|\cdot\|_{\infty}$ между указанными множествами, а константы Липшица берутся относительно $l^{1}$-нормы в евклидовом пространстве.

Для положительно и отрицательно ассоциированного случайного процесса или поля с конечными коэффициентами Кокса-Гримметта можно взять $\theta_{r}=u_{r}, r \in \mathbf{N}$ ([4, теорема 1.5.3]). Есть и другие важные примеры стохастических систем, удовлетворяющих определению $2[4, \S 1.5]$. Существенную роль в их описании (при условии стационарности) играет величина

$$
\sigma^{2}=\sum_{j \in \mathbf{Z}^{d}} \operatorname{cov}\left(X_{0}, X_{j}\right)
$$

называемая асимптотической или долгосрочной дисперсией случайного поля $X$. Она совпадает с дисперсией нормального распределения, которое возникает в центральной предельной теореме, если в качестве нормировки берется квадратный корень из числа слагаемых. Поэтому естественно задаться вопросом о статистическом оценивании величины $\sigma^{2}$ по наблюдениям $(B L, \theta)$-зависимого случайного поля.

Прежде всего введем ряд обозначений. Для векторов $a, b \in \mathbf{R}^{d}$ пишем $a \leqslant b$ (соответственно $a<b$ ), если $a_{i} \leqslant b_{i}$ (соответственно $a_{i}<b_{i}$ ) при $i=1, \ldots, d$, и при $a<b$ полагаем $(a, b]=\left\{t \in \mathbf{R}^{d}: a<t \leqslant b\right\}$. Также для $t=\left(t_{1}, \ldots, t_{d}\right) \in \mathbf{R}_{+}^{d}$ используем записи $t_{*}=\min _{i=1, \ldots, d} t_{i}$ и $|t|=t_{1} \ldots t_{d}$. Мощность конечного множества $U$ обозначаем $|U|$ (в частности, тогда $|n|=\left|(0, n] \cap \mathbf{Z}^{d}\right|, n \in \mathbf{N}^{d}$ ). Символ $n \rightarrow \infty$ означает, что $n_{*} \rightarrow \infty$. Все суммы вида $\sum_{j \leqslant b}$ берутся по $j \in \mathbf{N}^{d}, j \leqslant b$, если обратное не указано явно.

Пусть $X=\left\{X_{j}, j \in \mathbf{Z}^{d}\right\}$ - стационарное в узком смысле квадратичноинтегрируемое $(B L, \theta)$-зависимое случайное поле, а числа $\alpha, \beta>0, \alpha+\beta<1$. Предполагается, что случайное поле наблюдается на целочисленных параллелепипедах вида $(0, n] \cap \mathbf{Z}^{d}$, где $n \in \mathbf{N}^{d}$. Введем множества $Q_{j}^{(n)}, j \in \mathbf{N}^{d}$, полагая

$$
Q_{j}^{(n)}=\prod_{i=1}^{d}\left(\left(j_{i}-1\right)\left(\left[n_{*}^{\alpha}\right]+\left[n_{i} n_{*}^{-\beta}\right]\right) ; j_{i}\left[n_{*}^{\alpha}\right]+\left(j_{i}-1\right)\left[n_{i} n_{*}^{-\beta}\right]\right] \cap \mathbf{Z}^{d} .
$$

Пусть $T_{n}=\max \left\{j \in \mathbf{N}^{d}: Q_{j}^{(n)} \subseteq(0, n]\right\}$, где максимум понимается в смысле введенного выше частичного порядка в $\mathbf{R}^{d}$. Также нам потребуется $\Delta=\Delta_{n}$ для обозначения минимального расстояния между $Q_{j}^{(n)}$ и $Q_{k}^{(n)}$ при $j \neq k$ (расстояние берется в норме $\left.\|\cdot\|_{\infty}\right)$. Отметим, что при таком построении

$$
\left|Q_{j}^{(n)}\right| \sim n_{*}^{\alpha d}, \quad\left|T_{n}\right| \sim n_{*}^{\beta d}, \quad \Delta_{n} \sim n_{*}^{1-\beta} \quad \text { при } n \rightarrow \infty .
$$

Обозначая $S(U)=\sum_{j \in U} X_{j}$ для конечного $U \subset \mathbf{Z}^{d}$ и $S_{n}=S((0, n])$ при $n \in \mathbf{N}^{d}$, для каждого $s \in(0,1]$ рассмотрим статистики

$$
\widehat{\sigma_{s}}(n)=\frac{1}{\left|T_{n}\right|} \sum_{j \leqslant T_{n}}\left|Q_{j}^{(n)}\right|^{s / 2}\left|\frac{S\left(Q_{j}^{(n)}\right)}{\left|Q_{j}^{(n)}\right|}-\frac{S_{n}}{|n|}\right|^{s} .
$$


Подобные семейства статистик, названные статистиками с локальным усреднением, были введены М.Пелиград и К.-М.Шао [14] для систем с перемешиванием. Впоследствии значительные результаты, относящиеся к состоятельности таких оценок для ассоциированных полей, получены А. В. Булинским и М. А. Вронским [3], [4, §7.2]. Было показано, что при определенных условиях оценка (5) сходится в среднем должного порядка к числу $\sigma^{s} \mathbf{E}|Z|^{s}$, здесь и далее $Z \sim N(0,1)$. Это важное свойство и центральные предельные теоремы для зависимых случайных полей позволяют строить асимптотические доверительные интервалы для неизвестного математического ожидания поля $X$. Следует назвать также работы [2] и [8], где исследовались обобщения таких статистик на векторнозначные случайные поля. Целью данной заметки является установление условий асимптотической нормальности оценок с локальным усреднением. Отметим, что мы рассматриваем модификацию указанных статистик, так как важна слабая зависимость слагаемых в определении $\widehat{\sigma_{s}}(n)$. Начнем с доказательства сходимости распределений нормированных оценок к нормальному закону.

Теорема 1. Пусть $X$ - описанное выше случайное поле $u \theta_{r} \leqslant c_{0} r^{-\lambda}$ при некоторых $c_{0}, \lambda>0$ и всех $r \in \mathbf{N}$. Предположим, ито параметры $\alpha$ и $\beta$ удовлетворяют соотношениям

$$
\alpha s+\beta<s \quad u \quad \beta<\frac{\lambda s}{(1-s) d+\lambda s} .
$$

Тогда

$$
\sqrt{\left|T_{n}\right|}\left(\widehat{\sigma_{s}}(n)-\mathbf{E} \widehat{\sigma_{s}}(n)\right) \rightarrow Y \sim N\left(0, \sigma^{2 s} \mathbf{D}|Z|^{s}\right)
$$

по распределению, когда $n \rightarrow \infty$, здесь $Z \sim N(0,1)$.

Д о к а з а т е ль с т в о. Достаточно считать, что $\mathbf{E} X_{0}=0$. Сначала убедимся, что

$$
\frac{1}{\sqrt{\left|T_{n}\right|}} \sum_{j \leqslant T_{n}}\left|Q_{j}^{(n)}\right|^{s / 2} \frac{\left|S_{n}\right|^{s}}{|n|^{s}} \rightarrow 0
$$

в среднем, когда $n \rightarrow \infty$. В самом деле, применяя неравенство Ляпунова и элементарную оценку $\mathbf{E} S^{2}(U) \leqslant\left(\mathbf{E} X_{0}^{2}+\theta_{1}\right)|U|$, где конечное множество $U \subset \mathbf{Z}^{d}$, имеем

$$
\sqrt{\left|T_{n}\right|}\left|Q_{\mathbf{1}}^{(n)}\right|^{s / 2}|n|^{-s} \mathbf{E}\left|S_{n}\right|^{s} \leqslant C \sqrt{\left|T_{n}\right|}\left|Q_{\mathbf{1}}^{(n)}\right|^{s / 2}|n|^{-s / 2}
$$

(здесь и далее $C$ обозначает различные положительные множители, не зависящие от $n$, а $\left.\mathbf{1}=(1, \ldots, 1) \in \mathbf{N}^{d}\right)$. Ввиду (4) последнее выражение стремится к нулю при $n \rightarrow \infty$.

Из (8) и неравенства ||$x+\left.y\right|^{s}-\left.|x|^{s}|\leqslant| y\right|^{s}$, справедливого при всех $x, y \in \mathbf{R}$ и $s \in(0,1]$, следует, что достаточно проверить сходимость

$$
\frac{1}{\sqrt{\left|T_{n}\right|}} \sum_{j \leqslant T_{n}}\left(\frac{\left|S\left(Q_{j}^{(n)}\right)\right|^{s}}{\left|Q_{j}^{(n)}\right|^{s / 2}}-\mathbf{E} \frac{\left|S\left(Q_{j}^{(n)}\right)\right|^{s}}{\left|Q_{j}^{(n)}\right|^{s / 2}}\right) \rightarrow N\left(0, \sigma^{2 s} \mathbf{D}|Z|^{s}\right)
$$

по распределению, когда $n \rightarrow \infty$.

Лемма 1. Для любых $t_{1}, \ldots, t_{n} \in \mathbf{R}$ верна оченка

$$
\begin{gathered}
\left|\mathbf{E} \exp \left\{i \sum_{j \leqslant T_{n}} t_{j}\left|S\left(Q_{j}^{(n)}\right)\right|^{s}\right\}-\prod_{j \leqslant T_{n}} \mathbf{E} \exp \left\{i t_{j}\left|S\left(Q_{j}^{(n)}\right)\right|^{s}\right\}\right| \\
\leqslant C\left|T_{n}\right|\left|Q_{\mathbf{1}}^{(n)}\right|^{s /(2-s)} \theta_{\Delta}^{s /(2-s)} \max _{j=1, \ldots, n}\left|t_{j}\right|^{2 /(2-s)}
\end{gathered}
$$

где $\Delta$ фигурирует в (4).

Д о к а з а т е ль с т в о. Зададим $M>0$ и возьмем функцию $h_{M}$, определяемую равенством $h_{M}(x)=\max \{|x|, M\}^{s}, x \in \mathbf{R}$. Так как $\operatorname{Lip}\left(h_{M}\right)=s M^{s-1}$ и 
$\left.|| x\right|^{s}-h_{M}(x) \mid \leqslant M^{s}$ при $x \in \mathbf{R}$, то

$$
\begin{aligned}
& \left|\mathbf{E} \exp \left\{i \sum_{j \leqslant T_{n}} t_{j}\left|S\left(Q_{j}^{(n)}\right)\right|^{s}\right\}-\prod_{j \leqslant T_{n}} \mathbf{E} \exp \left\{i t_{j}\left|S\left(Q_{j}^{(n)}\right)\right|^{s}\right\}\right| \\
& \leqslant \sum_{j \in T_{n}} \mathbf{E}\left|\exp \left\{i t_{j}\left(\left|S\left(Q_{j}^{(n)}\right)\right|^{s}-h_{M}\left(S\left(Q_{j}^{(n)}\right)\right)\right)\right\}-1\right| \\
& \quad+\left|\mathbf{E} \exp \left\{i \sum_{j \leqslant T_{n}} t_{j} h_{M}\left(\left|S\left(Q_{j}^{(n)}\right)\right|\right)\right\}-\prod_{j \leqslant T_{n}} \mathbf{E} \exp \left\{i t_{j} h_{M}\left(\left|S\left(Q_{j}^{(n)}\right)\right|\right)\right\}\right| \\
& \leqslant\left|T_{n}\right| M^{s} \max _{j=1, \ldots, n}\left|t_{j}\right|+4\left|T_{n}\right|\left|Q_{1}^{(n)}\right| \theta_{\Delta} M^{2(s-1)} \max _{j=1, \ldots, n} t_{j}^{2} .
\end{aligned}
$$

Здесь в оценке суммы по $j \leqslant T_{n}$ мы воспользовались неравенством $\left|e^{i y}-1\right| \leqslant|y|$ (при $y \in \mathbf{R})$, а оценка последнего слагаемого следует из $(B L, \theta)$-зависимости и проверяется аналогично [4, следствие 1.5.5]. Остается минимизировать получившееся выражение по $M$. Лемма доказана.

Из леммы 1 мы получаем, что для каждого $t \in \mathbf{R}$

$$
\begin{aligned}
& \left|\mathbf{E} \exp \left\{i \frac{t}{\sqrt{\left|T_{n}\right|}} \sum_{j \in T_{n}} \frac{\left|S\left(Q_{j}^{(n)}\right)\right|^{s}}{\left|Q_{j}^{(n)}\right| s / 2}\right\}-\prod_{j \leqslant T_{n}} \mathbf{E} \exp \left\{i \frac{t}{\sqrt{\left|T_{n}\right|}} \frac{\left|S\left(Q_{j}^{(n)}\right)\right|^{s}}{\left|Q_{j}^{(n)}\right|^{s / 2}}\right\}\right| \\
& \leqslant C\left|T_{n}\right|^{(1-s) /(2-s)}|t|^{s /(2-s)} \theta_{\Delta}^{s /(2-s)} \\
& \leqslant C n_{*}^{(1-s) \beta d /(2-s)} n_{*}^{-\lambda(1-\beta) s /(2-s)} \rightarrow 0, \quad n \rightarrow \infty .
\end{aligned}
$$

В силу центральной предельной теоремы для $(B L, \theta)$-зависимых случайных полей [4, теорема 3.1.12] и теоремы 5.4 в [1] имеем

$$
\mathbf{D} \frac{\left|S\left(Q_{1}^{(n)}\right)\right|^{s}}{\left|Q_{\mathbf{1}}^{(n)}\right|^{s / 2}} \rightarrow \sigma^{2 s} \mathbf{D}|Z|^{s}
$$

при $n \rightarrow \infty$. Теперь соотношение (9) вытекает из (10), (11) и теоремы Линдеберга.

3 а м е ч а н и е 1 . Из первого условия на параметры $\alpha$ и $\beta$ следует, что $\beta<s$. Поэтому второе условие содержательно лишь при $s<1$ (при $s=1$ оно тривиально) и значениях $\lambda \leqslant d$.

$\mathrm{B}$ случае, когда $\sqrt{\left|T_{n}\right|}\left(\mathbf{E} \widehat{\sigma_{s}}(n)-\sigma^{s} \mathbf{E}|Z|^{s}\right) \rightarrow 0$ при $n \rightarrow \infty$, из теоремы 1 непосредственно следует, что

$$
\sqrt{\left|T_{n}\right|}\left(\mathbf{E} \widehat{\sigma_{s}}(n)-\sigma^{s} \mathbf{E}|Z|^{s}\right) \rightarrow Y
$$

по распределению, когда $n \rightarrow \infty$. Проверка указанного соотношения может быть проведена с помощью результатов типа Берри-Эссеена для $(B L, \theta)$-зависимых случайных полей. А именно, справедлива следующая лемма.

Лемма 2. Пусть случайное поле $X$ такое же, как в условиях теоремь 1 , причем $\sigma^{2}>0$ и $\mathbf{E}\left|X_{0}\right|^{2+\delta}<\infty, \delta \in(0,1]$. Тогда существует $C>0$ такое, ито для всех $n \in \mathbf{N}^{d}$ u $j \in \mathbf{Z}^{d}$ имеет место оценка

$$
\left.\left|\mathbf{E} \frac{\left|S\left(Q_{j}^{(n)}\right)\right|^{s}}{\left|Q_{j}^{(n)}\right|^{s / 2}}-\sigma^{s} \mathbf{E}\right| Z\right|^{s} \mid \leqslant C n_{*}^{-\nu},
$$

где $\nu=\delta \alpha d /\left(4+d(1+\delta)\left(4 \lambda^{-1}+6\right)\right)$.

Д о к а з а т е ль с т в о. Можно сразу считать, что $\sigma=1$. Согласно теореме 3.1 .21 из [4], справедлива оценка

$$
\sup _{x \in \mathbf{R}}\left|\mathbf{P}\left\{\frac{\left|S\left(Q_{j}^{(n)}\right)\right|}{\sqrt{\mathbf{D} S\left(Q_{j}^{(n)}\right)}}>x\right\}-\mathbf{P}\{|Z|>x\}\right| \leqslant C\left|Q_{j}^{(n)}\right|^{-\vartheta},
$$


где $\vartheta=\delta /\left(4+d(1+\delta)\left(4 \lambda^{-1}+6\right)\right)$. С другой стороны, согласно лемме 5.2.2, б из [4]

$$
\left|\mathbf{D} \frac{S\left(Q_{j}^{(n)}\right)}{\sqrt{\left|Q_{j}^{(n)}\right|}}-1\right| \leqslant C n_{*}^{-\lambda \alpha /(1+\lambda)} .
$$

Отсюда и из (13) следует, что

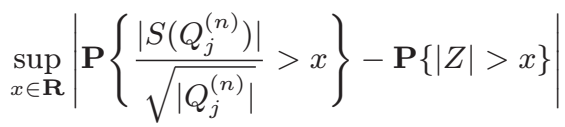

$$
\begin{aligned}
& =\sup _{x \in \mathbf{R}}\left|\mathbf{P}\left\{\frac{\left|S\left(Q_{j}^{(n)}\right)\right|}{\sqrt{\mathbf{D} S\left(Q_{j}^{(n)}\right)}}>x\right\}-\mathbf{P}\left\{|Z|>x \frac{\sqrt{\left|Q_{j}^{(n)}\right|}}{\sqrt{\mathbf{D} S\left(Q_{j}^{(n)}\right)}}\right\}\right| \\
& \leqslant C\left|Q_{j}^{(n)}\right|^{-\vartheta}+C n_{*}^{-\lambda \alpha /(1+\lambda)} \text {, }
\end{aligned}
$$

где мы воспользовались также оценкой [4, лемма 7.2.6]. Пользуясь (4) и теоремой 12 из $[5$, гл. $5, \S 5]$, имеем

$$
\begin{aligned}
\left.\left|\mathbf{E} \frac{\left|S\left(Q_{j}^{(n)}\right)\right|^{s}}{\left|Q_{j}^{(n)}\right|^{s / 2}}-\mathbf{E}\right| Z\right|^{s} \mid & \leqslant \int_{0}^{\infty} \mid \mathbf{P}\left\{\frac{\left|S\left(Q_{j}^{(n)}\right)\right|}{\left.\sqrt{\left|Q_{j}^{(n)}\right|}>x^{1 / s}\right\}-\mathbf{P}\left\{|Z|>x^{1 / s}\right\} \mid d x}\right. \\
& \leqslant C \int_{0}^{\infty} \frac{n_{*}^{-\vartheta \alpha d}+n_{*}^{-\lambda \alpha /(1+\lambda)}}{1+x^{2 / s}} d x
\end{aligned}
$$

откуда и следует утверждение леммы.

3 а м е ч а н и е 2. Из [11] известно, что уже для независимых одинаково распределенных центрированных слагаемых $\left\{\xi_{n}, n \in \mathbf{N}\right\}$ с конечным третьим моментом скорость сходимости первого абсолютного момента нормированной суммы $\mathbf{E}\left|\sum_{i=1}^{n} \xi_{i}\right| / \sqrt{n}$ к соответствующему моменту нормального распределения может иметь нижнюю оценку $n^{-c}$, где $c$ (при должном выборе распределения $\xi_{1}$ ) сколь угодно близко к $1 / 2$. Таким образом, в общем случае эта скорость сходимости не может быть существенно быстрее скорости сходимости распределений нормированных сумм к нормальному закону в метрике Колмогорова.

Следующая теорема дает построение асимптотического доверительного интервала для неизвестной долгосрочной дисперсии $\sigma^{2}$.

Теорема 2. Пусть выполнены условия теоремы 1 и леммы 2, причем $\beta d<2 \nu$, где $\nu$ введено в формулировке леммь 2. Тогда справедливо соотношение

$$
\sqrt{\left|T_{n}\right|}\left(\ln \frac{\widehat{\sigma_{s}}(n)}{\mathbf{E}|Z|^{s}}-s \ln \sigma\right) \rightarrow N\left(0, \frac{\mathbf{D}|Z|^{s}}{\left(\mathbf{E}|Z|^{s}\right)^{2}}\right)
$$

по распределению, когда $n \rightarrow \infty$.

Д о к а з а т е л ь с т в о. Из леммы 2 и теоремы 1 следует (12). Утверждение теоремы получается из (12) применением классического метода стабилизации дисперсии.

3 а м е ч а н и е 3 . Если в условиях теоремы 1 выполняется равенство $\sigma^{2}=$ 0 , то для справедливости соотношения (12) достаточно потребовать, чтобы $\beta d<$ $\alpha s \lambda /(1+\lambda)$. В самом деле, в этом случае (считая элементы случайного поля центрированными) имеем

$$
\sqrt{\left|T_{n}\right|}\left|Q_{\mathbf{1}}^{(n)}\right|^{-s / 2} \mathbf{E}\left|S\left(Q_{\mathbf{1}}^{(n)}\right)\right|^{s} \leqslant \sqrt{\left|T_{n}\right|}\left(\frac{\mathbf{D} S\left(Q_{\mathbf{1}}^{(n)}\right)}{\left|Q_{1}^{(n)}\right|}\right)^{s / 2} \leqslant C n_{*}^{\beta d / 2} n_{*}^{-\alpha \lambda s / 2(1+\lambda)} \rightarrow 0
$$

по лемме 5.2.2 [4]. Так как еще выполнено (8), то из приведенной оценки вытекает, что $\sqrt{\left|T_{n}\right|} \mathbf{E} \widehat{\sigma_{s}}(n) \rightarrow 0$. 
Известно [4, теорема 1.5.18], что стационарное гауссовское случайное поле с суммируемой ковариационной функцией является $(B L, \theta)$-зависимым. В качестве величин $\theta_{r}$ в этом случае можно взять коэффициенты

$$
\theta_{r}=\sum_{j:\|j\|_{\infty} \geqslant r}\left|\operatorname{cov}\left(X_{0}, X_{j}\right)\right|, \quad j \in \mathbf{N}
$$

аналогичные коэффициентам Кокса-Гримметта для положительно или отрицательно ассоциированных полей. Для гауссовского поля условия теорем 1 и 2 можно существенно упростить. А именно, справедлива следующая теорема.

Теорема 3. Пусть $X=\left\{X_{j}, j \in \mathbf{Z}^{d}\right\}$ - стационарное гауссовское случайное поле с абсолютно суммируемой ковариачионной функиией, причем $\sigma^{2}>0$, а оченки с локальным усреднением построены как в (5). Тогда

(а) справедливо соотношение (7);

(б) если к тому же $\beta d<2 \alpha \lambda /(1+\lambda)$, то выполняется $и$ (14).

Д о к а з а т е л ь с т в о. Начнем с двух лемм.

Лемма 3. Пусть $(\xi, \eta)-2$-мерный гауссовский случайный вектор с нулевым средним, $\mathbf{E} \xi^{2}=\mathbf{E} \eta^{2}=1$ и $\mathbf{E} \xi \eta=\rho \in(-1,1)$. Тогда при $s \in(0,1]$ верна оченка

$$
\mathbf{E}|\xi \eta|^{s-1} \leqslant G(s)(1-|\rho|)^{-1 / 2}
$$

где $G$ - некоторая функиия.

Д о к а з а т е л ь с т в о. Вычисляя математическое ожидание с помощью перехода к полярным координатам, имеем

$$
\begin{aligned}
\mathbf{E}|\xi \eta|^{s-1} & =\frac{1}{2 \pi \sqrt{1-\rho^{2}}} \iint_{\mathbf{R}^{2}}|x y|^{s-1} \exp \left\{-\frac{x^{2}+y^{2}-2 \rho x y}{2\left(1-\rho^{2}\right)}\right\} d y d x \\
& =\frac{1}{2 \pi \sqrt{1-\rho^{2}}} \int_{0}^{2 \pi}|\sin \varphi \cos \varphi|^{s-1} \int_{0}^{\infty} r^{2(s-1)+1} \exp \left\{-r^{2} \frac{1-\rho \sin 2 \varphi}{2\left(1-\rho^{2}\right)}\right\} d r d \varphi \\
& =\frac{2^{s}\left(1-\rho^{2}\right)^{s} \Gamma(s)}{2 \pi \sqrt{1-\rho^{2}}} \int_{0}^{2 \pi}|\sin \varphi \cos \varphi|^{s-1}(1-\rho \sin 2 \varphi)^{-s} d \varphi \\
& \leqslant \frac{2^{s-1} \Gamma(s)}{\pi}\left(1-\rho^{2}\right)^{s-1 / 2}(1-|\rho|)^{-s} \int_{0}^{2 \pi}|\sin \varphi \cos \varphi|^{s-1} d \varphi \\
& \leqslant \frac{2^{s} \Gamma(s)}{\pi}(1-|\rho|)^{-1 / 2} \int_{0}^{2 \pi}|\sin \varphi \cos \varphi|^{s-1} d \varphi .
\end{aligned}
$$

Остается заметить, что последний интеграл сходится.

Лемма 4. Пусть случайный вектор $\xi=\left(\xi_{1}, \ldots, \xi_{n}\right) \sim N(0, \Sigma)$, причем $\sigma_{j j}=\tau>$ $0(j=1, \ldots, n) u\left|\sigma_{1 j}\right|<\tau, j=2, \ldots, n$. Тогда для любых $t_{1}, \ldots, t_{n} \in \mathbf{R}$ верна оченка

$$
\begin{aligned}
& \left|\mathbf{E} \exp \left\{i \sum_{j=1}^{n} t_{j}\left|\xi_{j}\right|^{s}\right\}-\mathbf{E} \exp \left\{i t_{1}\left|\xi_{1}\right|^{s}\right\} \mathbf{E} \exp \left\{i \sum_{j=2}^{n} t_{j}\left|\xi_{j}\right|^{s}\right\}\right| \\
& \leqslant G(s) \tau^{s-1} \max _{j=1, \ldots, n}\left(1-\tau^{-1}\left|\sigma_{1 j}\right|\right)^{-1 / 2} \sum_{j=2}^{n}\left|t_{1} t_{j} \sigma_{1 j}\right| .
\end{aligned}
$$

Д о к а з а т е ль с т в о. Достаточно рассмотреть случай, когда матрица $\Sigma$ невырождена. Тогда общий случай получается предельным переходом от случайных векторов, имеющих матрицы ковариаций $\Sigma+m^{-1} I$, где $I$ - единичная матрица, $m \in$ $\mathbf{N}$ (см. завершение доказательства теоремы 1.2.1 [4]). Далее, сразу можно считать, что $\tau=1$. Пусть функции $f, g: \mathbf{R}^{n} \rightarrow \mathbf{C}$ определяются равенствами

$$
f(x)=\exp \left\{i t_{1}\left|x_{1}\right|^{s}\right\}, \quad g(x)=\exp \left\{i t_{2}\left|x_{2}\right|^{s}+\cdots+i t_{n}\left|x_{n}\right|^{s}\right\},
$$


здесь $x=\left(x_{1}, \ldots, x_{n}\right) \in \mathbf{R}^{n}$. Введем случайный вектор $\zeta \sim N(0, \Sigma)$, не зависящий от $\xi$, и положим

$$
F(\mu)=\mathbf{E} f(\xi) g\left(\mu \xi+\left(1-\mu^{2}\right)^{1 / 2} \zeta\right), \quad \mu \in[0,1],
$$

так что $F(1)-F(0)$ совпадает с выражением под знаком модуля в левой части (15). Пусть $\phi-$ плотность случайного вектора $\xi$ и $\phi_{\mu}(x)=\left(1-\mu^{2}\right)^{-n / 2} \phi\left(\left(1-\mu^{2}\right)^{-1 / 2} x\right)$, $\mu \in(0,1)$. Согласно лемме 1.2 .3 из [4], при $\mu \in(0,1)$ имеет место представление

$$
F^{\prime}(\mu)=\mu^{-1} \int_{\mathbf{R}^{n}} \phi(x) \sum_{j=2}^{n} \sigma_{1 j} \frac{\partial f(x)}{\partial x_{1}} \frac{\partial}{\partial x_{j}} \int_{\mathbf{R}^{n}} \phi_{\mu}(y) g(\mu x-y) d y d x .
$$

Точнее говоря, в условии указанной леммы в [4] требуется, чтобы функции $f$ и $g$ были непрерывны и ограничены вместе со всеми своими частными производными; однако ее утверждение несложно распространить на рассматриваемые функции, аппроксимируя функцию $x \mapsto e^{i t|x|^{s}}$ функциями из описанного класса и применяя теорему о мажорированной сходимости. Вычисляя производные, видим, что

$$
\begin{aligned}
& \left|F^{\prime}(\mu)\right| \leqslant \sum_{j=2}^{n}\left|\sigma_{1 j}\right| \int_{\mathbf{R}^{n}} \int_{\mathbf{R}^{n}} \phi(x) \phi_{\mu}(y)\left|x_{1}\right|^{s-1}\left|\mu x_{j}-y_{j}\right|^{s-1} d y d x \\
& =\sum_{j=2}^{n}\left|\sigma_{1 j}\right| \int_{\mathbf{R}^{n}} \int_{\mathbf{R}^{n}} \phi(x) \phi(z)\left|x_{1}\right|^{s-1}\left|\mu x_{j}-\left(1-\mu^{2}\right)^{1 / 2} z_{j}\right|^{s-1} d z d x \\
& =\sum_{j=2}^{n}\left|\sigma_{1 j}\right| \mathbf{E}\left|\xi_{1}\right|^{s-1}\left|\mu \xi_{j}-\left(1-\mu^{2}\right)^{1 / 2} \zeta_{j}\right|^{s-1} .
\end{aligned}
$$

Очевидно, случайный вектор $\left(\xi_{1}, \mu \xi_{j}-\left(1-\mu^{2}\right)^{1 / 2} \zeta_{j}\right)$ гауссовский с нулевым средним и единичными дисперсиями, причем $\left|\operatorname{cov}\left(\xi_{1}, \mu \xi_{j}-\left(1-\mu^{2}\right)^{1 / 2} \zeta_{j}\right)\right| \leqslant\left|\sigma_{1 j}\right|$, так что утверждение теперь следует из леммы 3 и формулы Лагранжа. Лемма 4 доказана.

Вернемся к доказательству теоремы 3. Обозначим для краткости

$$
\eta_{j}^{(n)}=\sqrt{\left|Q_{j}^{(n)}\right|}\left(\frac{S\left(Q_{j}^{(n)}\right)}{\left|Q_{j}^{(n)}\right|}-\frac{S_{n}}{|n|}\right), \quad j \in \mathbf{N}^{d} .
$$

Легко подсчитать, что

$$
\left|\operatorname{cov}\left(\eta_{j}^{(n)}, \eta_{k}^{(n)}\right)\right| \leqslant C\left(\frac{\left|\operatorname{cov}\left(S\left(Q_{j}^{(n)}\right), S\left(Q_{k}^{(n)}\right)\right)\right|}{\left|Q_{j}^{(n)}\right|}+\frac{\left|Q_{j}^{(n)}\right|}{|n|}\right), \quad j \neq k, \quad j, k \leqslant T_{n} .
$$

Также $\mathbf{D} \eta_{j}^{(n)} \rightarrow \sigma^{2}$ при $n \rightarrow \infty$. Используя лемму 4 , при всех $n$ таких, что $\mathbf{D} \eta_{j}^{(n)}>\sigma^{2} / 2$ и $\left|\operatorname{cov}\left(\eta_{j}^{(n)}, \eta_{k}^{(n)}\right)\right| \leqslant \sigma^{2} / 4$ (последнее неравенство - при $j \neq k$ ) мы имеем

$$
\begin{aligned}
& \left|\mathbf{E} \exp \left\{i \frac{t}{\sqrt{\left|T_{n}\right|}} \sum_{j \leqslant T_{n}} \eta_{j}^{(n)}\right\}-\prod_{j \leqslant T_{n}} \mathbf{E} \exp \left\{i \frac{t}{\sqrt{\left|T_{n}\right|}} \eta_{j}^{(n)}\right\}\right| \\
& \leqslant G(s)\left(\sigma^{2} / 2\right)^{s-1} 2^{1 / 2} t^{2} \max _{j \leqslant T_{n}} \sum_{k \leqslant T_{n}, k \neq j}\left|\operatorname{cov}\left(\eta_{j}^{(n)}, \eta_{k}^{(n)}\right)\right| \\
& \leqslant C\left(\theta_{\Delta}+\frac{\left|T_{n}\right|\left|Q_{j}^{(n)}\right|}{|n|}\right) \rightarrow 0
\end{aligned}
$$

при $n \rightarrow \infty$ (для второго слагаемого это следует из того, что $\alpha+\beta<1$ ). Из последнего соотношения первое утверждение теоремы выводится так же, как теорема 1 из леммы 1. Для проверки второго утверждения достаточно заметить, что

$$
\left.|\mathbf{E}| \eta_{j}^{(n)}\right|^{s}-\left.\mathbf{E}|Z|^{s} \sigma^{s}|=\mathbf{E}| Z\right|^{s}\left|\left(\mathbf{D}\left(\frac{S\left(Q_{j}^{(n)}\right)}{\sqrt{\left|Q_{j}^{(n)}\right|}}-\frac{\sqrt{\left|Q_{j}^{(n)}\right|} S_{n}}{|n|}\right)\right)^{s / 2}-\sigma^{s}\right|
$$




$$
\leqslant C\left(\frac{\left|Q_{j}^{(n)}\right|}{|n|}+\left|\mathbf{D} \frac{S\left(Q_{j}^{(n)}\right)}{\sqrt{\left|Q_{j}^{(n)}\right|}}-\sigma^{2}\right|\right), \quad j \in \mathbf{N}^{d} .
$$

Теперь из условий на $\alpha$ и $\beta$ и леммы 5.2.2 в [4] следует, что после умножения на $\sqrt{\left|T_{n}\right|}$ последнее выражение остается стремящимся к нулю при $n \rightarrow \infty$. Теорема доказана.

Функциональная предельная теорема. Перейдем к установлению функциональной предельной теоремы для оценок с локальным усреднением. Для этого положим $t b=\left(t_{1} b_{1}, \ldots, t_{1} b_{d}\right)$ при $t \in[0,1]^{d}, U \subseteq \mathbf{R}^{d}$ и введем случайные поля $\widehat{\sigma_{s}}(\cdot, n)$ с элементами

$$
\widehat{\sigma_{s}}(t, n):=\frac{1}{\left|T_{n}\right|} \sum_{j \leqslant t T_{n}}\left|Q_{j}^{(n)}\right|^{s / 2}\left|\frac{S\left(Q_{j}^{(n)}\right)}{\left|Q_{j}^{(n)}\right|}-\frac{S_{n}}{|n|}\right|^{s}, \quad t \in[0,1]^{d} .
$$

Условия справедливости функциональных предельных теорем для случайных элементов, принимающих значения в пространстве $L^{2}\left([0,1]^{d}\right)$, впервые рассматривались в фундаментальной статье Ю.В. Прохорова [6]. Впоследствии для зависимых (в том числе ассоциированных) процессов и полей многие результаты были получены П. Оливейрой и Ш. Сюке [12], [13].

Теорема 4. Пусть справедливь условия теоремь 1, причем вместо второго неравенства в (6) выполнено более сильное неравенство $\beta \leqslant \lambda s /(\lambda s+2 d(1-s))$. Тогда случайные прочессьи

$$
\left\{\sqrt{\left|T_{n}\right|}\left(\widehat{\sigma_{s}}(t, n)-\mathbf{E} \widehat{\sigma_{s}}(t, n)\right), t \in[0,1]^{d}\right\}, \quad n \in \mathbf{N}^{d},
$$

сходятся по распределению в пространстве $L^{2}\left([0,1]^{d}\right)$, когда $n \rightarrow \infty, \kappa$ случайному прочессу $\left(\mathbf{D}|Z|^{s}\right)^{1 / 2} \sigma^{s} W$, где $W-d$-параметрический винеровский прочесс. Если справедливы также условия теоремы 2 , то аналогичное утверждение верно и для прочессов $\left\{\sqrt{\left|T_{n}\right|}\left(\widehat{\sigma_{s}}(t, n)-\sigma^{s}|t| \mathbf{E}|Z|^{s}\right), t \in[0,1]^{d}\right\}_{n \in \mathbf{N}^{d}}$. Наконеи, в случае, когда поле $X$ гауссовское, для выполнения первого соотношения достаточно условий теоремь 3(а), а второго - условий теоремь 3(б).

Д о к а з а т е л ь с т в о. Пусть сначала рассматривается $(B L, \theta)$-зависимое случайное поле $X$. Проверим первое утверждение. Как и при доказательстве теоремы 1 , считаем поле $X$ центрированным. Тогда (см. (8)) $\sqrt{\left|T_{n}\right|}\left|Q_{1}^{(n)}\right|^{s / 2}\left|S_{n}\right|^{s} /|n|^{s} \rightarrow 0$ в среднем, когда $n \rightarrow \infty$. Поэтому вместо исходного случайного процесса достаточно рассмотреть процессы

$$
W_{n}(t)=\frac{1}{\left|T_{n}\right|} \sum_{j \leqslant t T_{n}} \frac{\left|S\left(Q_{j}^{(n)}\right)\right|^{s}-\mathbf{E}\left|S\left(Q_{j}^{(n)}\right)\right|^{s}}{\left|Q_{j}^{(n)}\right|^{s / 2}}, \quad n \in \mathbf{N}^{d} .
$$

Выберем в $L^{2}\left([0,1]^{d}\right)$ ортонормированный базис $\left\{e_{i}, i \in \mathbf{N}\right\}$ и положим $f_{i}(u)=$ $\int_{[u, \mathbf{1}]} e_{i}(t) d t, u \in[0,1]^{d}$ (берется обычное скалярное произведение в $L^{2}\left([0,1]^{d}\right)$ ). Тогда

$$
\begin{aligned}
\left(W_{n}, e_{i}\right) & =\frac{1}{\sqrt{\left|T_{n}\right|}} \int_{[0,1]^{d}} \sum_{j \leqslant t T_{n}} \frac{\left|S\left(Q_{j}^{(n)}\right)\right|^{s}-\mathbf{E}\left|S\left(Q_{j}^{(n)}\right)\right|^{s}}{\left|Q_{j}^{(n)}\right|^{s / 2}} e_{i}(t) d t \\
& =\frac{1}{\sqrt{\left|T_{n}\right|}} \sum_{j \leqslant T_{n}} \frac{\left|S\left(Q_{j}^{(n)}\right)\right|^{s}-\mathbf{E}\left|S\left(Q_{j}^{(n)}\right)\right|^{s}}{\left|Q_{j}^{(n)}\right| s / 2} f_{i}\left(j / T_{n}\right),
\end{aligned}
$$

где $j / m:=\left(j_{1} / m_{1}, \ldots, j_{d} / m_{d}\right)$ (для $\left.j, m \in \mathbf{N}^{d}\right)$. Следовательно, при $N \in \mathbf{N}$

$$
\sup _{n \in \mathbf{N}^{d}} \mathbf{E} \sum_{i=N}^{\infty}\left(W_{n}, e_{i}\right)^{2}=\sup _{n \in \mathbf{N}^{d}} \frac{1}{\left|T_{n}\right|} \sum_{j, k \leqslant T_{n}} a_{j k} \frac{\operatorname{cov}\left(\left|S\left(Q_{j}^{(n)}\right)\right|^{s},\left|S\left(Q_{k}^{(n)}\right)\right|^{s}\right)}{\left|Q_{j}^{(n)}\right|^{s}},
$$

где $a_{j k}=\sum_{i=N}^{\infty} f_{i}\left(j / T_{n}\right) f_{i}\left(k / T_{n}\right)$. В силу теоремы Дини и равенства Парсеваля ряд $\sum_{i} f_{i}^{2}(x)$ сходится равномерно по $x \in[0,1]^{d}$. 
Лемма 5. Для любого $j \in \mathbf{Z}^{d}$ имеет место оценка

$$
\sum_{k \leqslant T_{n}}\left|\operatorname{cov}\left(\left|S\left(Q_{j}^{(n)}\right)\right|^{s},\left|S\left(Q_{k}^{(n)}\right)\right|^{s}\right)\right| \leqslant C\left|Q_{j}^{(n)}\right|^{s} .
$$

Д о к а з а т е л ь с т в о. Прежде всего, $\mathbf{D}\left|S\left(Q_{j}^{(n)}\right)\right|^{s} \leqslant \mathbf{E}\left|S\left(Q_{j}^{(n)}\right)\right|^{2 s} \leqslant C\left|Q_{j}^{(n)}\right|^{s}$ по неравенству Ляпунова. Далее, каждому $k \leqslant T_{n}, k \neq j$, поставим в соответствие число $\gamma_{k}=\operatorname{sgn}\left(\operatorname{cov}\left(\left|S\left(Q_{j}^{(n)}\right)\right|^{s},\left|S\left(Q_{k}^{(n)}\right)\right|^{s}\right)\right)$. Пусть $M>0$ фиксировано и функция $h_{M}$ - та же, что использовалась при доказательстве леммы 1 . Тогда

$$
\begin{aligned}
\sum_{k \leqslant T_{n}, k \neq j}\left|\operatorname{cov}\left(\left|S\left(Q_{j}^{(n)}\right)\right|^{s},\left|S\left(Q_{k}^{(n)}\right)\right|^{s}\right)\right| \\
\quad=\operatorname{cov}\left(\left|S\left(Q_{j}^{(n)}\right)\right|^{s}, \sum_{k \leqslant T_{n}, k \neq j} \gamma_{k}\left|S\left(Q_{k}^{(n)}\right)\right|^{s}\right) \\
\quad \leqslant \operatorname{cov}\left(h_{M}\left(S\left(Q_{j}^{(n)}\right)\right), \sum_{k \leqslant T_{n}, k \neq j} \gamma_{k} h_{M}\left(S\left(Q_{k}^{(n)}\right)\right)\right)+2 M^{s} \mathbf{E}\left|S\left(Q_{j}^{(n)}\right)\right|^{s}\left|T_{n}\right| \\
\leqslant\left|Q_{j}^{(n)}\right| M^{2(s-1)} \theta_{\Delta}+2 M^{s} \mathbf{E}\left|S\left(Q_{j}^{(n)}\right)\right|^{s}\left|T_{n}\right|,
\end{aligned}
$$

где в последнем неравенстве мы использовали (2), а $\Delta$ фигурирует в (4). Остается найти минимум данного выражения по $M$ и заметить, что показатель степени при $n_{*}$ в получившемся выражении не превосходит $\alpha d s$. Лемма 5 доказана.

Из леммы 5 и (19) следует, что

$$
\begin{aligned}
\sup _{n \in \mathbf{N}^{d}} \mathbf{E} \sum_{i=N}^{\infty}\left(W_{n}, e_{i}\right)^{2} & \leqslant \sup _{x \in[0,1]^{d}} \sum_{i=N}^{\infty} f_{i}^{2}(x) \sup _{n \in \mathbf{N}^{d}} \max _{j \leqslant T_{n}} \sum_{k \leqslant T_{n}} \frac{\left|\operatorname{cov}\left(\left|S\left(Q_{j}^{(n)}\right)\right|^{s},\left|S\left(Q_{k}^{(n)}\right)\right|^{s}\right)\right|}{\left|Q_{j}^{(n)}\right|^{s}} \\
& \leqslant C \sum_{i=N}^{\infty} f_{i}^{2}(x) \rightarrow 0
\end{aligned}
$$

при $N \rightarrow \infty$. Таким образом (см. условие плотности в $\left[4\right.$, с. 289]), семейство $\left\{W_{n}\right\}_{n \in \mathbf{N}^{d}}$ плотно.

Для доказательства первого утверждения теоремы 4 теперь достаточно (см. [4, лемма 5.1.13]) убедиться, что для любой непрерывной функции $g:[0,1]^{d} \rightarrow \mathbf{R}$ из всюду плотного подмножества $L^{2}\left([0,1]^{d}\right)$ справедливо соотношение

$$
\left(W_{n}, g\right) \rightarrow\left(\mathbf{D}|Z|^{s}\right)^{1 / 2} \sigma^{s}(W, g)
$$

по распределению при $n \rightarrow \infty$. Правая часть (20) имеет гауссовское распределение с нулевым средним и дисперсией

$$
\left(\mathbf{D}|Z|^{s}\right) \sigma^{2 s} \int_{[0,1]^{d}} h^{2}(u) d u
$$

где функция $h$ определяется равенством

$$
h(u)=\int_{[u, \mathbf{1}]} g(t) d t, \quad t \in[0,1]^{d} .
$$

Левая же часть аналогично (18) преобразуется к виду

$$
\frac{1}{\sqrt{\left|T_{n}\right|}} \sum_{j \leqslant T_{n}} h\left(j / T_{n}\right) \frac{\left|S\left(Q_{j}^{(n)}\right)\right|^{s}-\mathbf{E}\left|S\left(Q_{j}^{(n)}\right)\right|^{s}}{\left|Q_{j}^{(n)}\right|^{s / 2}} .
$$

Для фиксированного $p \in \mathbf{N}$ разобьем куб $[0,1]^{d}$ на $p^{d}$ попарно непересекающихся кубов $B_{1}, \ldots, B_{p^{d}}$, каждый из которых имеет лебегову меру $p^{-d}$, и введем функцию $h_{p}:[0,1]^{d} \rightarrow \mathbf{R}$, постоянную на внутренности каждого из кубов $B_{1}, \ldots, B_{p^{d}}$ и такую, что ее значение в центральной точке каждого из этих кубов равно значению $h$ в этой точке. 
Лемма 6. Справедливо соотношение

$$
\frac{1}{\sqrt{\left|T_{n}\right|}} \sum_{j \leqslant T_{n}} h_{p}\left(j / T_{n}\right) \frac{\left|S\left(Q_{j}^{(n)}\right)\right|^{s}-\mathbf{E}\left|S\left(Q_{j}^{(n)}\right)\right|^{s}}{\left|Q_{j}^{(n)}\right|^{s / 2}} \rightarrow N\left(0,\left(\mathbf{D}|Z|^{s}\right) \sigma^{2 s} \int_{[0,1]^{d}} h_{p}^{2}(t) d t\right)
$$

по распределению, когда $n \rightarrow \infty$.

Д о к а з а т е л ь с т в о аналогично доказательству теоремы 1.

Так как функция $h$ липшицева, то, обозначая $V_{n}(h)$ левую часть $(21)$ и вводя аналогичную запись $V_{n}\left(h_{p}\right)$, имеем

$$
\begin{aligned}
\mathbf{E}\left(V_{n}(h)-V_{n}\left(h_{p}\right)\right)^{2} & \leqslant \operatorname{Lip}^{2}(h) p^{-2} \frac{1}{\left|T_{n}\right|} \sum_{j, k \leqslant T_{n}} \frac{\left|\operatorname{cov}\left(\left|S\left(Q_{j}^{(n)}\right)\right|^{s},\left|S\left(Q_{k}^{(n)}\right)\right|^{s}\right)\right|}{\left|Q_{j}^{(n)}\right|^{s}} \\
& \leqslant C \operatorname{Lip}^{2}(h) p^{-2}
\end{aligned}
$$

в силу леммы 5. Отсюда и из леммы 6 утверждение выводится стандартным образом (см. [4, лемма 5.1.14]).

Второе утверждение теоремы легко получить из первого и теоремы 2 , с учетом того, что

$$
\mathbf{E} \widehat{\sigma_{s}}(t, n)=\left|t T_{n}\right| \mathbf{E} \widehat{\sigma_{s}}(n) /\left|T_{n}\right|, \quad t \in[0,1]^{d}, \quad n \in \mathbf{N}^{d} .
$$

Теперь пусть поле $X$ - гауссовское. Доказательство теоремы в этом случае ведется аналогично приведенному выше (с теми же изменениями, которые были сделаны при доказательстве теоремы 3 по сравнению с теоремой 1). Единственное существенное отличие состоит в установлении аналога леммы 5. Именно, требуется убедиться, что при $j \leqslant T_{n}$

$$
\sum_{k \leqslant T_{n}}\left|\operatorname{cov}\left(\left|\eta_{j}^{(n)}\right|^{s},\left|\eta_{k}^{(n)}\right|^{s}\right)\right| \leqslant C
$$

где случайные величины $\eta_{j}^{(n)}$ введены в (16). Так как $\mathbf{D} \eta_{j}^{(n)} \rightarrow \sigma^{2}$ при $n \rightarrow \infty$, то существует такое $D>0$, что при всех $n$ таких, что $n_{*}>D$ выполняется неравенство $2 \sigma^{2} \geqslant \mathbf{D} \eta_{j}^{(n)} \geqslant \sigma^{2} / 2$ и, следовательно,

$$
\left|\operatorname{corr}\left(\eta_{j}^{(n)}, \eta_{k}^{(n)}\right)\right| \leqslant 2 \sigma^{-2}\left|\operatorname{cov}\left(\eta_{j}^{(n)}, \eta_{k}^{(n)}\right)\right|, \quad j \neq k, \quad j, k \leqslant T_{n} .
$$

По неравенству Гебелейна ([7, лемма 10.2$]$; в формулировке леммы по ссылке вместо $H$ следует читать $M)$ при $j \neq k$ имеем

$$
\begin{aligned}
\left|\operatorname{cov}\left(\left|\eta_{j}^{(n)}\right|^{s},\left|\eta_{k}^{(n)}\right|^{s}\right)\right| & \leqslant 2 \sigma^{-2}\left|\operatorname{cov}\left(\eta_{j}^{(n)}, \eta_{k}^{(n)}\right)\right| \mathbf{E}\left|\eta_{j}^{(n)}\right|^{2 s} \\
& \leqslant 2^{1+1 / s} \sigma^{-2+2 / s}\left|\operatorname{cov}\left(\eta_{j}^{(n)}, \eta_{k}^{(n)}\right)\right| .
\end{aligned}
$$

Так как справедлива оценка (17) и $\left|T_{n}\right|\left|Q_{j}^{(n)}\right| /|n| \rightarrow 0$ при $n \rightarrow \infty$, то (22) теперь вытекает из суммируемости ковариационной функции случайного поля.

Автор признателен профессору А. В. Булинскому за внимание к работе и полезные обсуждения.

\section{СПИСОК ЛИТЕРАТУРЫ}

1. Биллингсли П. Сходимость вероятностных мер. М.: Наука, 1977, 352 с.

2. Булинский $A . B$. Статистический вариант центральной предельной теоремы для векторнозначных случайных полей. - Матем. заметки, 2004, т. 76, № 4, с. 490501.

3. Булинский A. В., Вронский М. А. Статистический вариант центральной предельной теоремы для ассоциированных случайных полей. - Фундам. и прикл. матем., 1996, т. 2, № 4, с. 999-1018. 
4. Булинский A. В., Шашкин А. П. Предельные теоремы для ассоциированных случайных полей и родственных систем. М.: ФИЗМАТЛИТ, 2008, 477 с.

5. Петров В.В. Предельные теоремы для сумм независимых случайных величин. М.: Наука, 1987, 317 с.

6. Прохоров Ю.В. Сходимость случайных процессов и предельные теоремы теории вероятностей. - Теория вероятн. и примен., 1956, т. 1, в. 2, с. 177-238.

7. Розанов Ю.А. Стационарные случайные процессы. М.: Наука, 1990, 284 с.

8. Bulinski A., Kryzhanovskaya $N$. Convergence rate in CLT for vector-valued random fields with self-normalization. - Probab. Math. Stat., 2006, v. 26, № 2, p. 261-281.

9. Bulinski A., Suquet Ch. Normal approximation for quasi-associated random fields. Statist. Probab. Lett., 2001, v. 54, № 2, p. 215-226.

10. Esary J., Proschan F., Walkup D. Association of random variables, with applications. - Ann. Math. Statist., 1967, v. 38, № 5, p. 1466-1474.

11. Hall $P$. Bounds on the rate of convergence of moments in the central limit theorem. Ann. Probab., 1982, v. 10, № 4, p. 1004-1018.

12. Oliveira P.E., Suquet Ch. An invariance principle in $L^{2}[0,1]$ for nonstationary $\varphi$ mixing sequences. - Comment. Math. Univ. Carol., 1995, v. 36, p. 293-302.

13. Oliveira P.E., Suquet Ch. An $L^{2}[0,1]$ invariance principle for LPQD random variables. - Portugaliae mathematica, 1996, v. 53, № 2, p. 367-379.

14. Peligrad M., Shao Q.-M. A note on estimation of variance for $\rho$-mixing sequences. Stat. Probab. Lett., 1996, v. 26, № 2, p. 141-145.

15. Prakasa Rao B.L. Associated Sequences, Demimartingales and Nonparametric Inference. Boston: Birkhäuser, 2012, 272 p.

Поступила в редакцию

5.XII.2013

(c) 2014 г.

ШВЕДОВ А. С.*

\section{МАТРИЧНОЕ $\boldsymbol{t}$-РАСПРЕДЕЛЕНИЕ С ВЕКТОРОМ СТЕПЕНЕЙ СВОБОДЫ}

В работе вводятся матричные $t$-распределения, для которых параметр, представляющий число степеней свободы, является многомерным. Получена формула для плотности распределения.

Ключевые слова и фразы: $t$-распределение случайной матрицы, плотность распределения.

1. Введение. Различные варианты многомерных $t$-распределений подробно рассматриваются, например, в книге [8]. Многомерные $t$-распределения широко используются в приложениях.

Матричные $t$-распределения изучаются, например, в [7, гл. 4] и применяются, в частности, при байесовском анализе регрессионных моделей (см., например, [1]). Эти распределения являются обобщением многомерных $t$-распределений. Однако в предшествующих нашим исследованиях тот параметр, который для одномерных $t$ распределений называется числом степеней свободы, и для многомерных $t$-распределений, и для матричных $t$-распределений остается положительным числом.

Матричные $t$-распределения тесно связаны с матричными гамма-распределениями (которые используются и в этой работе). Именно многомерный параметр в матричном гамма-распределении обусловливает появление многомерного параметра в матричном $t$-распределении.

* Национальный исследовательский университет «Высшая школа экономики», Москва, Россия; e-mail: ashvedov@hse.ru 